\title{
Évolution des acides aminés et de la matière organique dissoute dans une filière de production d'eau potable: Corrélations avec le carbone organique dissous biodégradable et le potentiel de demande en chlore à long terme Evolution of Amino Acids and Dissolved Organic Matter in a Drinking Water Treatment Plant: Correlations with Biodegradable Dissolved Organic Carbon and Long-Term Chlorine Demand
}

\author{
F. Dossier-Berne, N. Merleti, B. Cauchi et B. Legube
}

Volume 9, numéro 1, 1996

URI : https://id.erudit.org/iderudit/705245ar

DOI : https://doi.org/10.7202/705245ar

Aller au sommaire du numéro

Éditeur(s)

Université du Québec - INRS-Eau, Terre et Environnement (INRS-ETE)

ISSN

0992-7158 (imprimé)

1718-8598 (numérique)

Découvrir la revue

Citer cet article

Dossier-Berne, F., Merleti, N., Cauchi, B. \& Legube, B. (1996). Évolution des acides aminés et de la matière organique dissoute dans une filière de production d'eau potable: Corrélations avec le carbone organique dissous biodégradable et le potentiel de demande en chlore à long terme. Revue des sciences de l'eau / Journal of Water Science, 9(1), 115-133.

https://doi.org/10.7202/705245ar
Résumé de l'article

Le suivi de la matière organique totale (COD) et spécifique (substances humiques et principalement acides aminés dissous totaux) dans les eaux d'une filière de production d'eau potable a mis en évidence des abattements moyens dans l'eau traitée représentant $40 \%$ du COD et 0 à $75 \%$ des acides aminés. Parallèlement, le potentiel de demande en chlore à long terme est diminué de près de $70 \%$, alors que la fraction biodégradable connait une élimination de l'ordre de $50 \%$.

Aucune corrélation directe n'a pu être mise en évidence entre les teneurs en acides aminés et la valeur du carbone organique dissous biodégradable (CODB) et de la demande en chlore des eaux brutes ou en cours de traitement. Cependant, compte tenu des teneurs en acides aminés dissous totaux quantifiées dans les eaux traitées de l'usine, leur participation peut être estimée entre 5 et $23 \%$ du potentiel de demande en chlore et entre 5 et $25 \%$ du CODB.

La matière organique analysée spécifiquement dans le cadre de cette étude (substances humiques et acides aminés dissous totaux), représente 20 à $35 \%$ du COD des eaux en fin de filière, et la présence de ces composés organiques serait responsable d'une part notable de la demande en chlore à long terme des eaux (40 à $60 \%$ ). 


\title{
Evolution des acides aminés et de la matière organique dissoute dans une filière de production d'eau potable : corrélations avec le carbone organique dissous biodégradable et le potentiel de demande en chlore à long terme
}

\author{
Evolution of amino acids and dissolved organic matter \\ in a drinking water treatment plant : correlations \\ with biodegradable dissolved organic carbon \\ and long-term chlorine demand
}

F. DOSSIER BERNE1, N. MERLET ${ }^{1, *}$, B. CAUCH ${ }^{2}$ et B. LEGUBE ${ }^{1}$

Reçu le 21 octobre 1994, accepté le 6 juin 1995*.

\section{SUMMARY}

Dissolved organic compounds (dissolved organic carbon, DOC, biodegradable dissolved organic carbon, BDOC, total amino acids and humic substances) and their chlorine demand were analyzed monthly at different steps of the water treatnuent plant of Méry-sur-Oise (Paris, France $-270000 \mathrm{~m}^{3} / \mathrm{d}$, fig. I).

Total dissolved amino acids were deternined by HPLC analysis with fluorimetric detection after hydrolysis of combined amino acids (proteins, polypeptides) by hydrochloric acid (DOSSIER BERNE et al., 1994a); the separation of 17 amino acids was performed after orthophthaldialdehyde (OPA) pre-column derivatization. Humic substances were determined according to the method developed by THURMAN and MALCOLM (1981), by gravity feeding XAD-8 resins with acidified samples. The humic fraction was expressed as the difference between DOC before and after passage through the column. The method developed by JORET and LÉVI (1986) using a biologically active sand inoculum was used for the determination of BDOC.

For the determination of chlorine consumption, the kinetic model described by JADAS-HÉCART et al. (1992) was used; it takes into account the long-term chlorine demand in terms of potential demand. This method was automated (DOSSIER BERNE et aL, 1994b) and computer-assisted. The chlorine dose was chosen depending on the $\mathrm{DOC}$ value and on the $\mathrm{N}-\mathrm{NH}_{4}{ }^{+}$content of the water ; the applied dose was: $3 \mathrm{mg} \mathrm{Cl} / \mathrm{mg} \mathrm{DOC}+10 \mathrm{mg} \mathrm{Cl} / \mathrm{mg} \mathrm{N}-\mathrm{NH}_{4}{ }^{+}$. Chlorine determination was performed automatically by the spectrophotometric $\mathrm{N}$, $\mathrm{N}$-diethylphenylene-1,4-diamine (DPD) method.

1. Laboratoire de Chimie de l'Eau et des Nuisances, URA CNRS 1468. Ecole Supérieure d'Ingénieurs de Poitiers, 40, avenue du Recteur Pineau, 86022 Poitiers Cedex, France.

2. Anjou Recherche, Centre de Recherche de la Compagnie Générale des Eaux. Chemin de la Digue, BP 76, 78603 Maisons Laffitte, France.

* Les commentaires seront reçus jusqu'au 30 août 1996. 
In raw water, DOC values may reach 5.6 to $6.5 \mathrm{mg} \mathrm{C1}^{-1}$ during the cold season, but the average yield of elimination was generally close to $40 \%$ (fig. 2 ). The biodegradable fraction of the organic carbon (BDOC), which represents 25 to $50 \%$ of the DOC in the raw water, was partially removed in the plant and the residual concentration in treated water varied between 0.4 to $1.8 \mathrm{mg} \mathrm{C}-1$ (fig. 2). A transitory increase in the BDOC values was generally observed during the ozonation step; it reached 0.2 to $0.5 \mathrm{mg} \mathrm{Ct}^{-1}$ (figs. 6 and 7).

Whatever the period of the year, extracted humic substances constituted about $50 \%$ of the DOC found in raw water (fig. 3). This hydrophobic fraction was significantly remoged in the treatment plant reducing the proportion of humic substances in the DOC of treated water to $16-23 \%$ (figs. 3, 6 and 7).

The concentrations of total dissolved amino acids ranged from 100 to $260 \mu \mathrm{g}^{-1} \mathrm{C}$ in raw water and from 50 to $150 \mu \mathrm{g} \mathrm{I}^{-1} \mathrm{C}$ in produced water (fig. 4); the main part of this elimination occurred during the clarification step (figs. 6 and 7). No important seasonal variations could be observed for chlorine demand (fig. 5 ); its removal occurred at each step of treatment and the average global elimination by the plant was of the order of $70 \%$ (figs. 6 and 7 ).

On account of the low concentrations of amino acids, no direct relation could be shown between amino acid concentrations and the respective values of $\mathrm{BDOC}$ or of chlorine demand potential (figs. 9 and 10). With regards to BDOC and chlorine demand potential, no correlation could be shown between these two parameters either (fig. 8). However, it was possible to calculate the contribution of specific classes of dissolved organic compounds to chlorine demand and to BDOC. This calculation is based: i) on the concentrations of humic substances and amino acids measured during the monthly experiments at each point of the treatment plant, ii) on bibliographic data concerning the contribution to BDOC (biodegradability) and chlorine consumption of a large variety of model molecules (free or combined amino acids, aquatic humic substances).

The results obtained in this way are reported in Table 3 for the treated water of the Méry-sur-Oise plant. These results indicate that the small amounts of total dissolved amino acids present in treated water may account for 5 to $25 \%$ of the BDOC value and for 5 to $23 \%$ of the total chlorine demand potential. With regard to humic substances, their biodegradability was assumed to be very low but their contribution to the chlorine demand of the treated water was estima. ted between 10 to $40 \%$, whereas their DOC contribution ranges from 16 to $35 \%$. As a consequence, both amino acids and humic substances could account for 40 to $60 \%$ to the chlorine demand of treated water. A special point should be noticed for total amino acids: their contribution to the DOC values of treated water was only 2 to $7 \%$, but they may account for a larger proportion of BDOC or chlorine demand potential (5 to $25 \%$ ).

Key words : drinking water, treatment plant, amino acids, chlorine demand, BDOC.

Le suivi de la matière organique totale (COD) et spécifique (substances humiques et principalement acides aminés dissous totaux) dans les eaux d'une filière de production d'eau potable a mis en évidence des abattements moyens dans l'eau traitée représentant $40 \%$ du COD et 0 à $75 \%$ des acides aminés. Parallèlement, le potentiel de demande en chlore à long terme est diminué de près de $70 \%$, alors que la fraction biodégradable connait une élimination de l'ordre de $50 \%$.

Aucune corrélation directe n'a pu être mise en évidence entre les teneurs en acides aminés et la valeur du carbone organique dissous biodégradable (CODB) et de la demande en chlore des eaux brutes ou en cours de traitement. Cependant, compte tenu des teneurs en acides aminés dissous totaux quanti- 
fiées dans les eaux traitées de l'usine, leur participation peut être estimée entre 5 et $23 \%$ du potentiel de demande en chlore et entre 5 et $25 \%$ du CODB.

La matière organique analysée spécifiquement dans le cadre de cette étude (substances humiques et acides aminés dissous totaux), représente 20 à $35 \%$ du COD des eaux en fin de filière, et la présence de ces composés organiques serait responsable d'une part notable de la demande en chlore à long terme des eaux $(40$ à $60 \%)$.

Mots clés : eau potable, filière de traitement, acides aminés, demande en chlore, CODB.

\section{GLOSSAIRE}

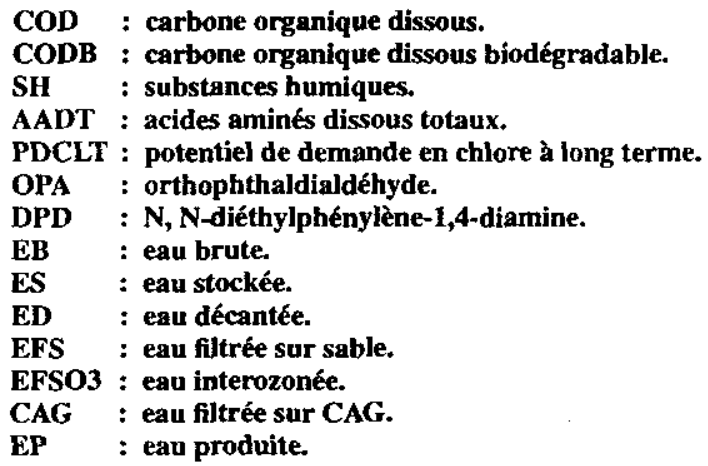

\section{1 - INTRODUCTION}

Dans les usines de traitement d'eau potable, l'évaluation des procédés de traitement et le contrôle de la qualité de l'eau traitée sont basés sur la détermination de très nombreux paramètres; parmi ceux-ci, le carbone organique dissous (COD) et le carbone organique dissous biodégradable (CODB), la demande en chlore à court terme ( 2 ou 4 heures), ou encore la formation de sous-produits de désinfection.

Une meilleure connaissance des composés spécifiques biodégradables et/ou consommateurs de chlore permettrait d'optimiser le traitement par l'élimination de la fraction biodégradable du COD et la réduction de la demande en chlore.

Des études récentes ont ainsi mis en évidence que certaines classes de composés (substances humiques, sucres, acides aminés) jouaient un rôle non négligeable dans l'évolution de la qualité bactériologique de l'eau en réseau de distribution, du fait de leur bonne aptitude à la biodégradation ou de leur forte demande en chlore.

- Les substances humiques représentent en moyenne 30 à $60 \%$ du COD des eaux naturelles (LEGUBE et al., 1990). Considérées comme généralement réfractaires à la biodégradation (LEENHEER et al., 1989), leur demande en chlore serait comprise entre 0,8 et $2 \mathrm{mg} \mathrm{Ct_{2 }} / \mathrm{mg} \mathrm{C}$ (RECKHOW, 1984 ; LEGUBE et al., 1990). 
- Les sucres participent faiblement au COD des eaux naturelles $(1 \%$ pour les saccharides et disaccharides, 5 à $10 \%$ pour les polysaccharides d'après THURMAN, 1985) et présentent vraisemblablement une consommation en chlore négligeable (JADAS-HÉCART, 1989 ; CRANE et al. 1980). Ils sont considérés comme moyennement biodégradables avec un ratio moyen CODB/COD de $0,5 \mathrm{mg} \mathrm{C} / \mathrm{mg}$ $C$ déterminés par AGBEKODO (1994).

- Les acides aminés sont présents dans les eaux naturelles à de très faibles concentrations de quelques dizaines à quelques centaines de $\mathrm{nmol} \mathrm{I}^{-1}$, soit 1 à $10 \%$ du COD (THURMAN, 1985 ; DOSSIER BERNE et al., 1994a), mais ils ont la caractéristique d'être à la fois facilement biodégradables et fortement consommateurs de chlore.

Cette aptitude à la biodégradation est confirmée par les travaux de ROTH et MAIER (1991), de MERLET et al. (1991) et d'AGBEKODO (1994). Ce dernier, travaillant sur 14 acides aminés libres et 3 acides aminés combinés, a mis en évidence des rapports moyens CODB/COD respectivement de 0,76 et 0,63 . Selon ce même auteur, ces résultats permettraient d'attribuer aux acides aminés une participation de $40 \%$ à la valeur du CODB dans une eau clarifiée.

En ce qui concerne la consommation en chlore des acides aminés, HUREIKI (1993) a montré que leur demande en chlore sur 72 heures variait suivant leur nature de 2,5 à $16,5 \mathrm{mg} \mathrm{Cl} / \mathrm{mg} \mathrm{C}$ (en moyenne 5 à $7 \mathrm{mg} \mathrm{Cl} / \mathrm{mg} \mathrm{C}$ ) dans le cas d'acides aminés libres, et de 2,5 à $4 \mathrm{mg} \mathrm{Cl} / \mathrm{mg} \mathrm{C}$ dans le cas d'acides aminés combinés. Pour ces derniers, la consommation en chlore peut se prolonger sur plusieurs centaines d'heures (EL MORER, 1992). D'après JADAS-HÉCART (1989), les acides aminés seraient à l'origine de 20 à $30 \%$ du potentiel total de demande en chlore de l'eau produite par l'usine de Choisy le Roi à partir de l'eau de la Seine (banlieue de Paris). Ces valeurs sont confirmées par AGBEKODO (1994) qui considère que $25 \%$ de la demande en chlore de l'eau filtrée sur sable de Mérysur-Oise pourraient être attribués à ces mêmes composés.

Au regard de la bibliographie, les acides aminés apparaissent donc comme un paramètre de choix pour évaluer la qualité d'une eau traitée, tant sur le plan de sa stabilité biologique que sur le plan de sa réactivité aux oxydants.

L'étude entreprise a consisté à mettre en œuvre sur une usine de traitement d'eau potable une vaste campagne analytique en vue d'établir une relation entre la consommation en chlore d'une eau, son CODB et sa teneur en certains composés organiques spécifiques (substances humiques et principalement acides aminés dissous totaux). Le support de cette étude a été l'usine de traitement de Méry-sur-Oise (banlieue de Paris, France). Les campagnes se sont déroulées à une fréquence mensuelle de novembre 1992 à novembre 1993.

\section{2 - MATÉRIELS ET MÉTHODES}

\subsection{Eaux étudiées}

L'usine de Méry-sur-Oise implantée sur l'Oise à une quinzaine de kilomètres en amont de sa confluence avec la Seine, alimente en eau potable 600000 habitants de la banlieue Nord de Paris. Elle a une capacité de production de 
$270000 \mathrm{~m}^{3} / j$ our. La figure 1 schématise les traitements appliqués ainsi que les points de prélèvement des échantillons. Des échantilions sont prélevés chaque mois sur l'ensemble de ces points, de novembre 1992 à novembre 1993.

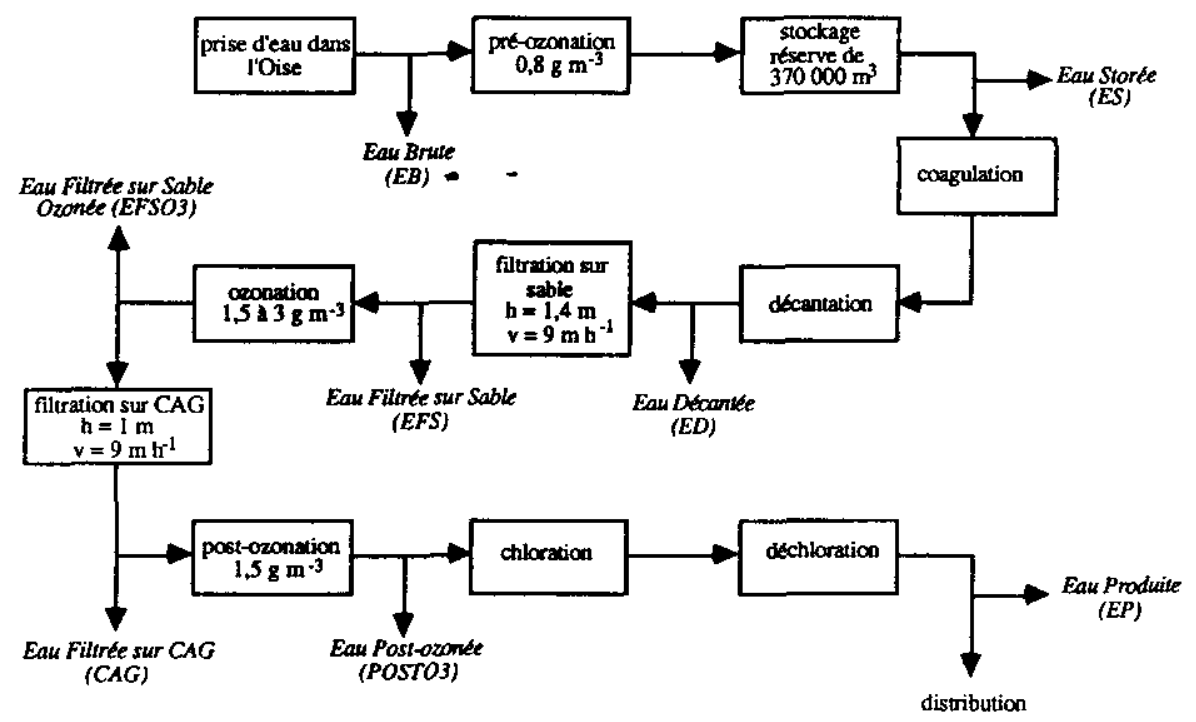

Figure 1 Schéma du procédé de traitement appliqué à l'usine de Méry-sur-Oise.

Treatment processes at the water treatment plant of Méry-sur-Oise.

\subsection{Méthodes analytiques}

\subsubsection{Procédure générale (nettoyage de la verrerie)}

La verrerie est soigneusement nettoyée au détergent Extran (Merck) dilué au $1 / 20$, puis rincée à l'eau Milli-Q (produite à partir d'une unité Millipore Milli-RO4 et Milii-Q) présentant une résistivité de $1810^{6} \Omega \mathrm{cm}$ et une teneur en COT inférieure à $0,1 \mathrm{mg} \mathrm{t}^{-1}$. Une partie (flacons de prélèvement, tubes pour COT, verrerie pour le dosage des acides aminés,...) est passée au four à $550^{\circ} \mathrm{C}$ pendant 4 heures de façon à éliminer toute trace de matière organique. La verrerie utilisée pour la détermination de la demande en chlore est stockée sous une solution de chlore (100 $\mathrm{mg} \mathrm{t}^{-1} \mathrm{Cl}_{2}$ ), puis rincée à l'eau Milli-Q avant utilisation. Lors du dosage des acides aminés, des gants sont utilisés pour éviter une contamination des échantillons lors des manipulations. Les prélèvements sont effectués dans des flacons en verre munis de bouchons à joints téflon et conservés à $4^{\circ} \mathrm{C}$ avant analyse (24 à 72 heures maximum).

\subsubsection{Analyse du COD}

Les échantillons sont préalablement filtrés sur filtre Millex HV de 0,45 $\mu \mathrm{m}$ (Millipore). 
La mesure du COD est réalisée à l'aide d'un analyseur de carbone Dohrmann DC 80 fonctionnant avec ou sans injecteur automatique. Chaque échantillon est injecté au moins 3 fois et la moyenne est effectuée sur des valeurs présentant un écart inférieur à $0,1 \mathrm{mg} \mathrm{I}^{-1} \mathrm{C}$.

\subsubsection{Détermination du CODB}

Cette mesure est basée sur la méthode décrite par JORET et LÉVI (1986) avec un inoculum de bactéries fixées sur sable. Toutes les analyses ont été réalisées au Laboratoire Central de la Compagnie Générale des Eaux (Paris, France).

\subsubsection{Détermination de la teneur en substances humiques}

L'analyse des teneurs en substances humiques (SH) a été obtenue en utilisant la méthode développée par THURMAN et MALCOLM (1981) et recommandée par l'International Humic Substances Society. L'échantillon d'eau, tout d'abord filtré sur membranes de $10 \mu \mathrm{m}$ puis $0,45 \mu \mathrm{m}$, est acidifié à pH 2 . L'échantillon acidifié percole ensuite sur une résine XAD-8 préalablement purifiée. Selon ce protocole, la matière organique dissoute retenue sur la résine $X A D-8$ à $\mathrm{pH}$ acide constitue la fraction humique. Une élution à la soude permet ensuite l'extraction de ces substances humiques.

\subsubsection{Dosage des acides aminés dissous totaux}

L'analyse est réalisée par chromatographie liquide haute performance (CLHP) avec détection fluorimétrique après dérivation précolonne à l'orthophthaldialdéhyde (OPA). Le protocole analytique suivi est décrit en détail par DOSSIER BERNE et al. (1994a). Les échantillons d'eau, préalablement filtrés sur filtre Millex HV $0,45 \mu \mathrm{m}$ et séchés sous vide, sont hydrolysés par $\mathrm{HCl} 6 \mathrm{~N}$ à $120^{\circ} \mathrm{C}$ pendant 3 heures (module Picotag Waters). La dérivation est réalisée par l'injecteur automatique AS 4000 Merck avant injection. Le réactif dérivant est constitué d'un mélange OPA-mercaptoéthanol-tampon borate. La séparation est assurée sur une colonne C18 Delta Pak Waters thermostatée à $30^{\circ} \mathrm{C}$ en utilisant un gradient d'élution (pompe System Controller 600E Waters) entre un tampon acétate-phosphate $(50 \mathrm{mM}, \mathrm{pH}=7,4)$ et un mélange méthanol-eau (75-25). La détection est assurée par un spectrofluorimètre F-1050 Merck à une longueur d'onde d'excitation de $335 \mathrm{~nm}$ et d'émission de $425 \mathrm{~nm}$. Le logiciel (Baseline 810 Waters) permet l'acquisition et le traitement des chromatogrammes.

Chaque échantillon est traité 5 fois et un calcul de moyenne est effectué. Les résultats sont exprimés en $\mathrm{nmol} \mathrm{I}^{-1}$ pour les teneurs individuelles avec une incertitude de 5 à $20 \%$ et en $\mu \mathrm{g}^{-1}$ d'azote ou de carbone pour les teneurs globales avec une incertitude de 5 à $15 \%$.

\subsubsection{Détermination automatisée de la demande en chiore à long terme}

La consommation en chlore des eaux est déterminée en terme de potentiel de consommation à long terme par une méthode automatisée (DOSSIER BERNE et al., 1994b) en utilisant l'interprétation cinétique du modèle développée par JADAS-HÉCART et al. (1992). Cet auteur divise la courbe de consommation en chlore en 2 parties :

- une première phase de consommation rapide sur 4 heures, appelée demande initiale ; 
- une phase de consommation lente s'étalant sur plusieurs centaines d'heures ou consommation à long terme, dont la vitesse peut être exprimée par une loi cinétique du second ordre (ordre 1 par rapport au substrat, ordre 1 par rapport au chlore).

La valeur de l'asymptote vers laquelle tend cette courbe est le potentiel de demande en chlore à long terme (PDCLT).

Les doses de chlore appliquées sont fonction du COD et de la teneur en azote ammoniacal (présent uniquement dans les eaux brutes, stockées et décantées). Les taux appliqués sont de $3 \mathrm{mg} \mathrm{Cl} / \mathrm{mg} C O D$ auxquels sont ajoutés $10 \mathrm{mg}$ $\mathrm{Cl}_{2} / \mathrm{mg} \mathrm{N} \mathrm{NH}_{4}{ }^{+}$, quand la concentration en ion ammonium est supérieure à $0,02 \mathrm{mg} \mathrm{t}^{-1}$. Les échantillons chlorés sont placés sur un analyseur automatique pour le suivi au cours du temps de la teneur en chlore libre résiduel. La mesure du chlore libre est basée sur la norme AFNOR T90-038 (1987), qui concerne le dosage du chlore libre et du chlore total par la méthode colorimétrique à la $N$, $\mathrm{N}$-dièthylphénylène-1,4-diamine (DPD). Le système se compose d'un automate modèle 222 Gilson muni d'un diluteur modèle 401 Gilson, couplé à un spectrophotomètre Secomam (S 1000 PC). La mesure d'absorbance est effectuée à une longueur d'onde de $510 \mathrm{~nm}$. Le pilotage du spectrophotomètre ainsi que l'enregistrement des absorbances mesurées se font par l'intermédiaire du logiciel $S 1000$ PC de Secomam.

\section{3 - RÉSULTATS ET DISCUSSION}

Pendant une année, l'ensemble des paramètres analytiques présentés ci-dessus a été suivi à chaque étape de la filière (fig. 1). En raison du grand nombre de résultats recueillis, il a été délibérément choisi de n'en présenter qu'une partie, c'est à dire :

- l'évolution saisonnière des acides aminés dissous totaux, de la matière organique et de la demande en chlore sur deux types d'eaux, l'eau brute et l'eau traitée,

- l'évolution de ces mêmes paramètres lors du traitement sur la filière et ceci uniquement sur deux mois de l'année (représentatifs de l'hiver et de l'été).

Des corrélations prenant en compte l'ensemble des résultats seront tentées entre les paramètres demande en chlore, CODB et acides aminés dissous totaux.

\section{1 Évolution saisonnière de la matière organique dissoute et du potentiel de demande en chlore dans les eaux brutes et traitées}

Les évolutions de la matière organique dissoute (COD, CODB, SH, AADT) et de sa réactivité avec le chiore sont rapportées pour les eaux brutes et traitées aux figures 2 à 5 et au tableau 1 .

La teneur en matière organique totale (COD) est relativement élevée dans l'eau brute ; le plus souvent comprise entre 3 à $4,2 \mathrm{mg} \mathrm{l}^{-1} \mathrm{C}$, elle présente des pointes en saison froide atteignant 5,6 et $6,5 \mathrm{mg} \mathrm{l}^{-1} \mathrm{C}$ (fig. 2). Dans une rivière 


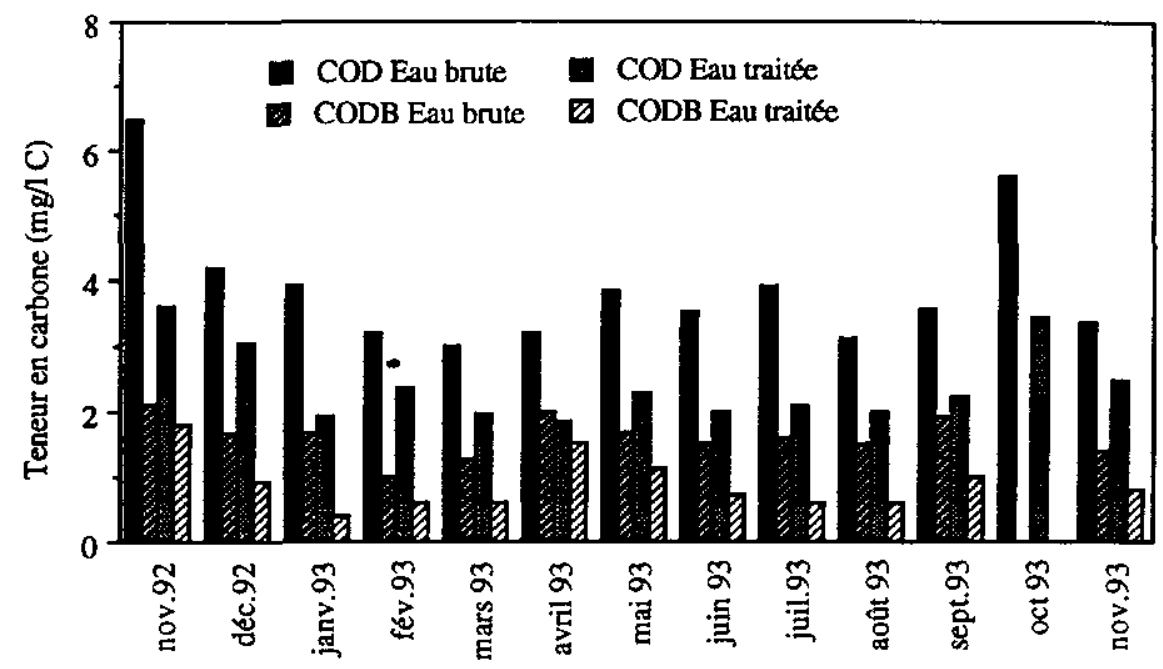

Figure 2 Evolution saisonnière du COD et du CODB dans les eaux brutes et traitées de la fillière de Méry-sur-Oise.

Seasonal evolution of $D O C$ and BDOC for raw and treated water in the treatment plant of Méry-sur-Oise.

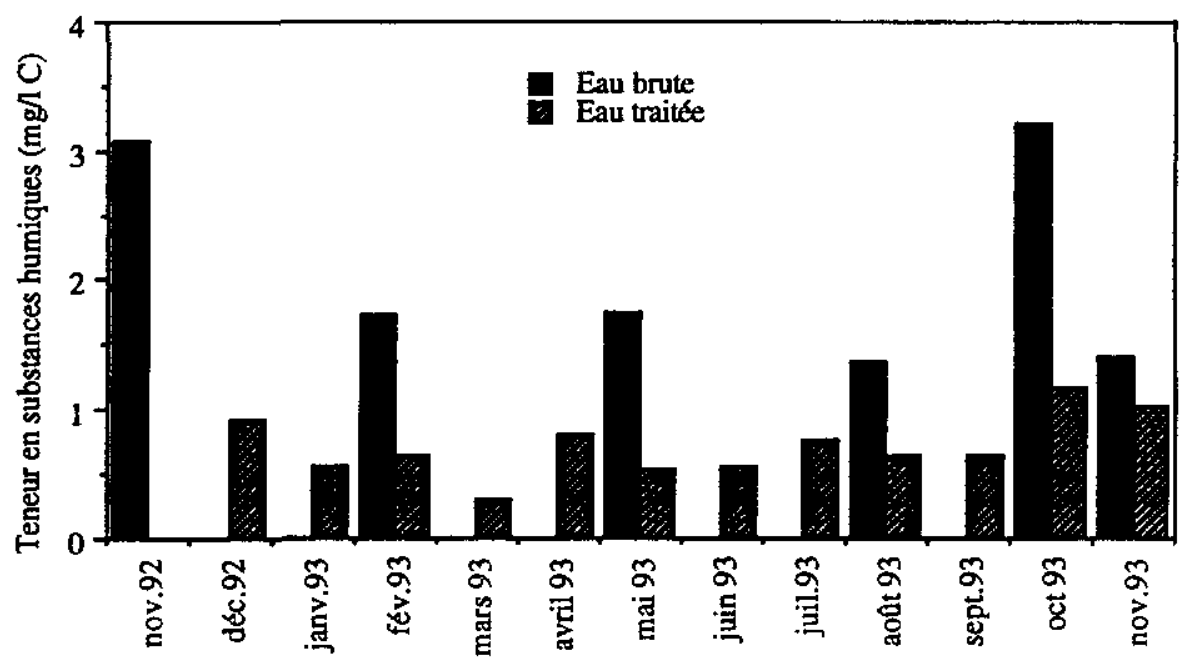

Figure 3 Evolution saisonnière de la teneur en substances humiques dans les eaux brutes et traitées de la filière de Méry-sur-Oise.

Seasonal evolution of the humic substance content for raw and treated water in the treatment plant of Méry-sur-Oise. 


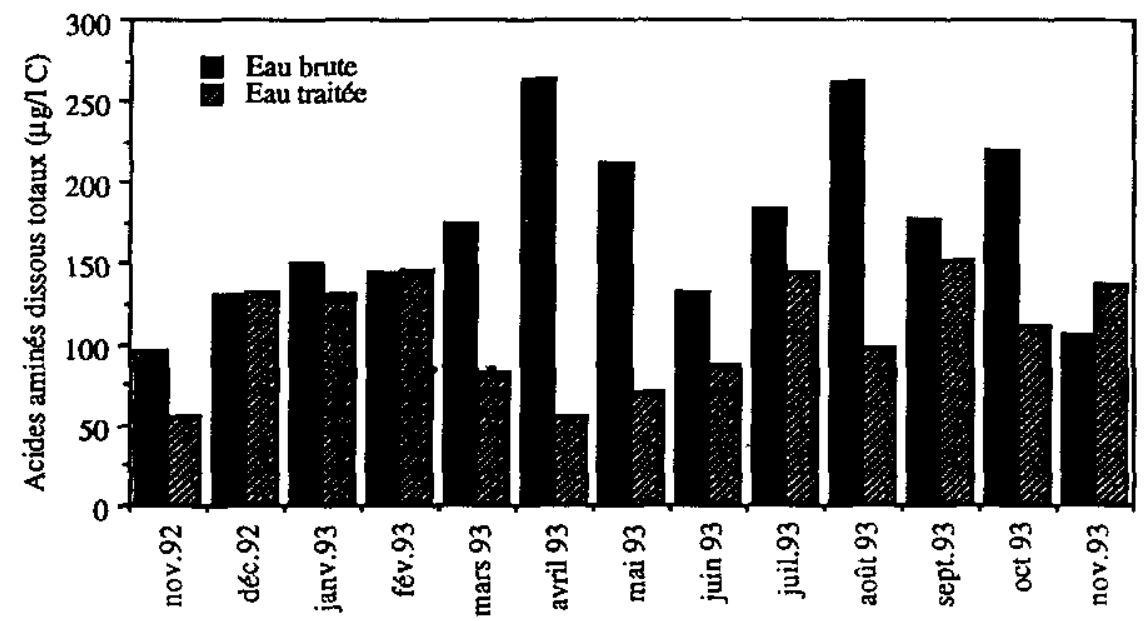

Figure 4 Evolution saisonnière de la teneur en acides aminés dissous totaux dans les eaux brutes et traitées de la filière de Méry-sur-Oise.

Seasonal evolution of the total dissolved amino acids for raw and treated water in the treatment plant of Méry-sur-Oise.

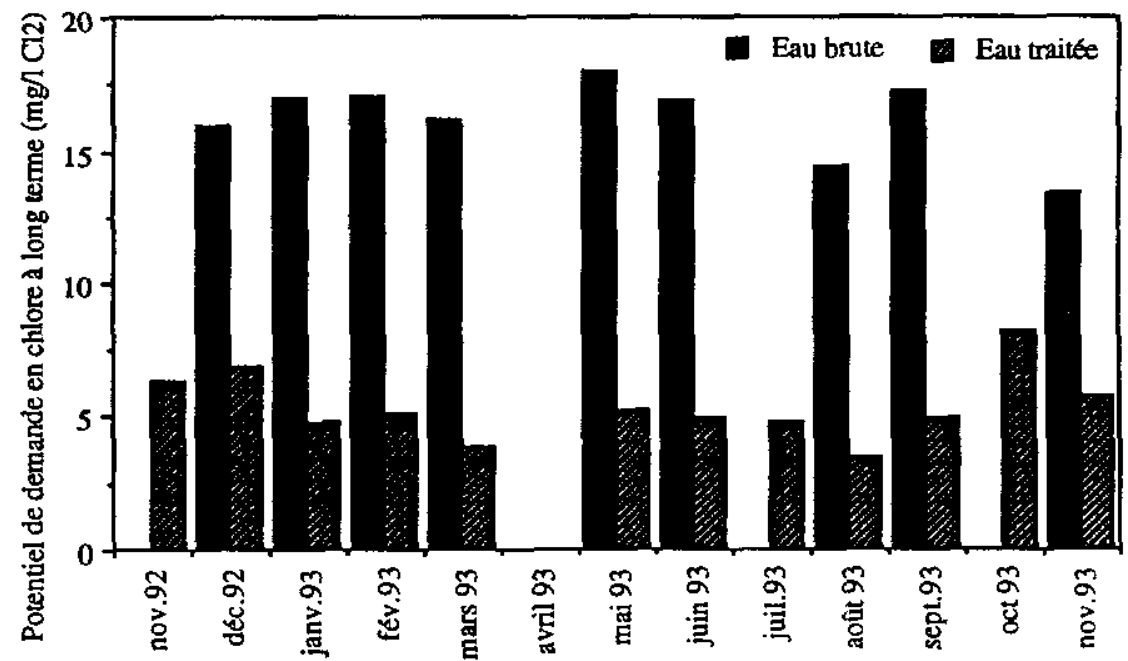

Figure 5 Evolution saisonnière du potentiel de demande en chlore à long terme dans les eaux brutes et traitées de la filière de Méry-sur-Oise.

Seasonal evolution of the long-term chlorine demand potential for raw and treated water in the treatment plant of Méry-sur-Oise. 
telle que l'OISE, les variations du COD dans l'eau brute sont le fait pour partie de la saison, mais elles reflètent également d'autres facteurs comme la pluviométrie. En moyenne, le COD de l'eau brute est de $3,9 \mathrm{mg} \mathrm{t}^{-1} \mathrm{C}$. Dans l'eau traitée, des variations similaires à celles de l'eau brute sont observées et la teneur moyenne en COD est de $2,4 \mathrm{mg} \mathrm{I}^{-1} \mathrm{C}$. L'abattement en COD semble relativement constant au cours de l'année avec un taux d'élimination moyen de $40 \%$.

L'élimination du CODB sur la filière de traitement n'est pas toujours correcte (fig. 2). Elle permet de passer de teneurs comprises entre 1 et $2,1 \mathrm{mg}^{-1} \mathrm{C}(1,6$ en moyenne) dans l'eau brute à des teneurs de 0,4 à $1,8 \mathrm{mg}^{-1} \mathrm{C}(0,7$ en moyenne) dans l'eau traitée. ta part du CODB dans le COD de l'eau produite varie ainsi de 20 à $48 \%$, ce qui reste relativement élevé. La concentration en CODB dans l'eau brute est peu sujette à des variations saisonnières contrairement à l'eau traitée.

La fraction de substances humiques $\left(1,4\right.$ à $\left.3,2 \mathrm{mg} \mathrm{l}^{-1} \mathrm{C}\right)$, qui constitue dans l'eau brute de Méry-sur-Oise près de la moitié du COD (42 à $53 \%$ ), est partiellement éliminée en cours de traitement (fig. 3). Elle ne représente plus dans l'eau produite que des teneurs en carbone variant de 0,3 à $1,2 \mathrm{mg} \mathrm{I}^{-1} \mathrm{C}$, soit 16 à $35 \%$ du COD résiduel. Bien que l'analyse des substances humiques n'ait pas été réalisée pour l'ensemble des échantillonnages, on peut remarquer que les teneurs en substances humiques analysées dans l'eau brute présentent les mêmes variations saisonnières que le $C O D$, ce qui conduit à une participation relativement constante en cours d'année.

Pour les acides aminés dissous totaux, leurs concentrations varient de 100 à $260 \mu \mathrm{g} \mathrm{I}^{-1} \mathrm{C}$ (soit 30 à $90 \mu \mathrm{g} \mathrm{I}^{-1} \mathrm{~N}$ ) dans l'eau brute et de 50 à $150 \mathrm{\mu g} \mathrm{I}^{-1} \mathrm{C}(20$ à $60 \mu \mathrm{g} \mathrm{I}^{-1} \mathrm{~N}$ ) dans l'eau produite (fig. 4). L'influence saisonnière est très marquée pour ce type de composé. En effet, on observe des teneurs maximales dans l'eau brute pendant le printemps et l'été (4,5 à $9 \%$ du COD). Les teneurs en acides aminés de l'eau traitée sont, à l'inverse, maximales durant l'hiver, l'abattement sur la filière étant alors quasiment nul. Au printemps et en été, les teneurs dans l'eau traitée sont notablement inférieures à celles de l'eau brute, ce qui témoigne d'une élimination comprise entre 20 et $75 \%$. Une légère augmentation de la teneur en acides aminés a parfois été observée dans les eaux traitées ; elle résulterait vraisemblablement d'une décomplexation par ozonation de structures complexes contenant des acides aminés, ce qui conduirait à les rendre accessible à l'hydrolyse et donc à l'analyse.

Les valeurs de potentiel de demande en chlore dans l'eau brute sont élevées (13,5 à $18 \mathrm{mg}^{1-1} \mathrm{Cl}_{2}$ ) (fig. 5); elles s'expliquent d'une part, par les fortes teneurs en COD et par les concentrations élevées en azote ammoniacal $\left(0,3 \mathrm{à} 0,6 \mathrm{mg}^{-1}\right)$ d'autre part. Elles semblent peu marquées par la période de prélèvement. Dans l'eau traitée, les demandes en chlore varient de 3,5 à $8 \mathrm{mg} \mathrm{I}^{-1} \mathrm{Cl}_{2}$, soit un abattement moyen le long de la filière de $70 \%$.

\section{2 Évolution de la matière organique dissoute et de la demande en chlore dans la filière de traitement}

L'évolution de la matière organique dissoute (COD, CODB, AADT, SH) et de sa réactivité au chlore sont rapportées à titre d'exemple pour les mois de décembre 1992 et août 1993 aux figures 6 et 7 et au tableau 2. 
Tableau 1 Evolution saisonnière de la matière organique dissoute et du potentiel de dernande en chlore à long terme dans les eaux brutes et traitées de la filière de Méry-sur-Oise. Table 1 Seasonal evolution of dissolved organic matter for raw and treated water in the trealment plant of Mery-sur-Oise.

\begin{tabular}{|c|c|c|c|c|c|c|c|c|c|c|}
\hline & \multicolumn{5}{|c|}{ Eau brut: } & \multicolumn{5}{|c|}{ Eau traitée } \\
\hline & $\underset{\mathrm{mg}^{-1} \mathrm{COD}}{\mathrm{C}}$ & $\begin{array}{c}\operatorname{CODB} \\
m g^{-1} \mathrm{C}\end{array}$ & $\frac{\mathrm{SH}}{\mathrm{mg} \mathrm{l}^{-1} \mathrm{C}}$ & $\underset{\mu \mathrm{I}^{-1} \mathrm{C}}{\mathrm{AADT}}$ & $\underset{\mathrm{mg}^{-1} \mathrm{Cl}_{2}}{\mathrm{PDCLT}}$ & $\underset{m g^{-1} \mathrm{C}}{\mathrm{COD}}$ & $\begin{array}{c}\mathrm{CODB} \\
\mathrm{mg} \mathrm{t}^{-1} \mathrm{C}\end{array}$ & $\underset{m g}{S H} H^{-1} \mathrm{C}$ & $\begin{array}{c}\text { AADT } \\
\mu 0^{-1} C\end{array}$ & 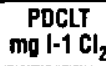 \\
\hline $\begin{array}{l}\text { Novembre } 1992 \\
\text { Oécembre } 1992 \\
\text { Janvier } 1993 \\
\text { Février } 1993 \\
\text { Mars } 1993 \\
\text { Avril } 1993 \\
\text { Mai } 1993 \\
\text { Jein } 1993 \\
\text { Juillet } 1993 \\
\text { Août } 1993 \\
\text { Septembre } 1993 \\
\text { Octobre } 1993 \\
\text { Novembre } 1993\end{array}$ & $\begin{array}{l}6,5 \\
4,2 \\
3,9 \\
3,2 \\
3,0 \\
3,2 \\
3,8 \\
3,5 \\
3,9 \\
3,1 \\
3,6 \\
5,6 \\
3,4\end{array}$ & $\begin{array}{l}2,1 \\
1,7 \\
1,7 \\
1,0 \\
1,3 \\
2,0 \\
1,7 \\
1,5 \\
1,6 \\
1,5 \\
1,9 \\
1,4\end{array}$ & $\begin{array}{c}3,1 \\
\vdots \\
1,7 \\
\vdots \\
\vdots \\
\vdots \\
1,4 \\
3,2 \\
1,4\end{array}$ & $\begin{array}{r}97 \\
131 \\
151 \\
141 \\
175 \\
264 \\
213 \\
132 \\
184 \\
263 \\
178 \\
220 \\
105\end{array}$ & $\begin{array}{c}>18 \\
16,0 \\
17,0 \\
17,1 \\
16,1 \\
\vdots \\
17,9 \\
16,9 \\
>18 \\
14,4 \\
17,3 \\
>18 \\
13,4\end{array}$ & $\begin{array}{l}3,4 \\
2,9 \\
1,9 \\
2,5 \\
1,8 \\
1,8 \\
2,1 \\
2,4 \\
2,2 \\
1,7 \\
2,2 \\
3,5 \\
2,3\end{array}$ & $\begin{array}{l}1,8 \\
0,9 \\
0,4 \\
0,6 \\
0,6 \\
1,5 \\
1,1 \\
0,7 \\
0,6 \\
0,6 \\
1,0 \\
0,8\end{array}$ & $\begin{array}{l}0,9 \\
0,6 \\
0,6 \\
0,3 \\
0,8 \\
0,6 \\
0,6 \\
0,8 \\
0,7 \\
0,7 \\
1,2 \\
1,0\end{array}$ & \begin{tabular}{r|}
56 \\
133 \\
131 \\
146 \\
83 \\
56 \\
71 \\
88 \\
145 \\
98 \\
153 \\
112 \\
137
\end{tabular} & $\begin{array}{l}6,3 \\
6,9 \\
4,7 \\
5,1 \\
3,8 \\
5 \\
5,3 \\
4,9 \\
4,7 \\
3,5 \\
4,9 \\
8,2 \\
5,7\end{array}$ \\
\hline
\end{tabular}

* Analyse non effectuée.

Tableau 2 Evolution sur la filière de Méry-sur-Oise de la matière organique dissoute et du potentiel de demande en chiore à long terme pour les mois de décembre 1992 er aoùt 1993. Table 2 Evolution of dissolved organic matter and of long-term chlorine demand potential in the water treatment plant of Mery-sur-Oise (December 1992 and August 1993).

\begin{tabular}{|c|c|c|c|c|c|c|c|c|c|c|}
\hline & & & cembre 19 & & & & & Août 1993 & & \\
\hline & $\mathrm{mg} \mathrm{F}^{-1} \mathrm{c}$ & $\begin{array}{c}\mathrm{CODB} \\
\mathrm{mg} \mathrm{l}^{-1} \mathrm{C}\end{array}$ & $\frac{\mathrm{SH}}{\mathrm{mg} \mathrm{F}^{-1} \mathrm{C}}$ & $\begin{array}{c}\text { AADT } \\
\mu \mathrm{I}^{-1} \mathrm{C}\end{array}$ & $\begin{array}{c}\text { PDCLT } \\
\mathrm{mg}^{-1} \mathrm{Cl}_{2}\end{array}$ & $\begin{array}{c}\mathrm{coO} \\
\left.\operatorname{mg}\right|^{-1} \mathrm{C}\end{array}$ & $\begin{array}{c}\mathrm{COOB} \\
\mathrm{mg} \mathrm{l}^{-1} \mathrm{C}\end{array}$ & $\begin{array}{c}\mathrm{SH} \\
\mathrm{mg} \mathrm{r}^{-1} \mathrm{C}\end{array}$ & $\begin{array}{c}\text { AADT } \\
\mu g r^{-1} \mathrm{C}\end{array}$ & $\begin{array}{c}\text { PDCLT } \\
\mathrm{mg} /-1 \mathrm{Cl}\end{array}$ \\
\hline$E B$ & 4,2 & 1,7 & * & 131 & 16,0 & 3,1 & 1,5 & 1,4 & 263 & 14,4 \\
\hline ES & 4,9 & 1,5 & 2,3 & 165 & 16,6 & 3,0 & 1,2 & 1,2 & 164 & 13,6 \\
\hline$E D$ & 4,3 & 1,0 & 2,0 & 91 & 10,8 & 2,9 & 0,8 & 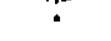 & 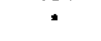 & 10,3 \\
\hline EFS & 3,9 & 0,6 & . & 74 & 9,1 & 2,4 & 0,8 & 0,9 & 100 & 6,4 \\
\hline EFS03 & 3,7 & 1,0 & - & 88 & 8,2 & 2,1 & 0,8 & $*$ & 76 & 4,8 \\
\hline CAG & 2,9 & 0,6 & , & 98 & 6,9 & 1,7 & 0,6 & * & 85 & 3,5 \\
\hline EP & 3,1 & 0,9 & 0,9 & 133 & * & 2,0 & 0,6 & 0,7 & 98 & 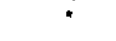 \\
\hline
\end{tabular}

- Analyse non effectuée. 


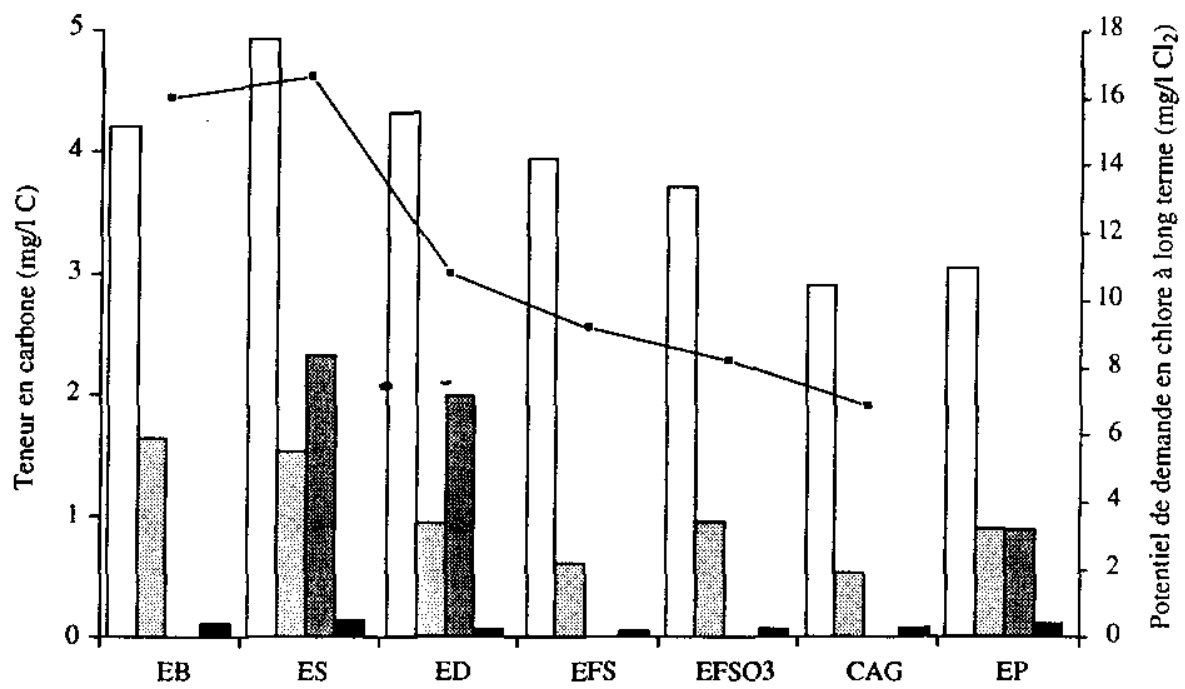

Figure 6 Evolution sur la filière de Méry-sur-Oise de la matière organique dissoute (COD, CODB, SH, AADT) et de sa demande en chlore pour le mois de décembre 1992.

Evolution of dissolved organic compounds (DOC, BDOC, Humic Substances and Total Dissolved Amino Acids) and of chlorine demand potential in the water treatment plant of Méry-sur-Oise (December 1992)

Si la diminution des valeurs des divers paramètres semble s'opérer progressivement le long de la filière de traitement, des différences significatives apparaissent selon le paramètre considéré et selon le procédé de traitement concerné.

Au regard de l'ensemble des résultats obtenus sur l'année, la clarification (coagulation-floculation-décantation-filtration sur sable) semble être une des principales étapes responsables des abattements observés. En effet, si toutes les étapes de traitement sont impliquées dans l'élimination de la demande en chlore, avec une évolution parallèle à celle du COD, une élimination de l'ordre de $60 \%$ est en général observée lors de la clarification. Au cours des traitements ultérieurs la diminution du potentiel total de demande en chlore est beaucoup plus faible. Globalement, l'abattement de la demande en chlore à long terme peut atteindre $70 \%$.

De même, l'essentiel de l'élimination des acides aminés dissous totaux se produit apparemment lors des premières étapes de traitement (stockage, clarification). Certains auteurs attribuent cette élimination des acides aminés, très solubles dans l'eau, à l'étape de coagulation/floculation, par leur adsorption à la surface des flocs formés (LE CLOIREC et al., 1983). A l'inverse, l'étape de clarification ne semble pas conduire à une élimination préférentielle des substances humiques (fig. 7), alors que ces composés en raison de leurs masses molaires élevées sont présumés être facilement éliminables (LEFEBVRE et LEGUBE, 1990 ; 


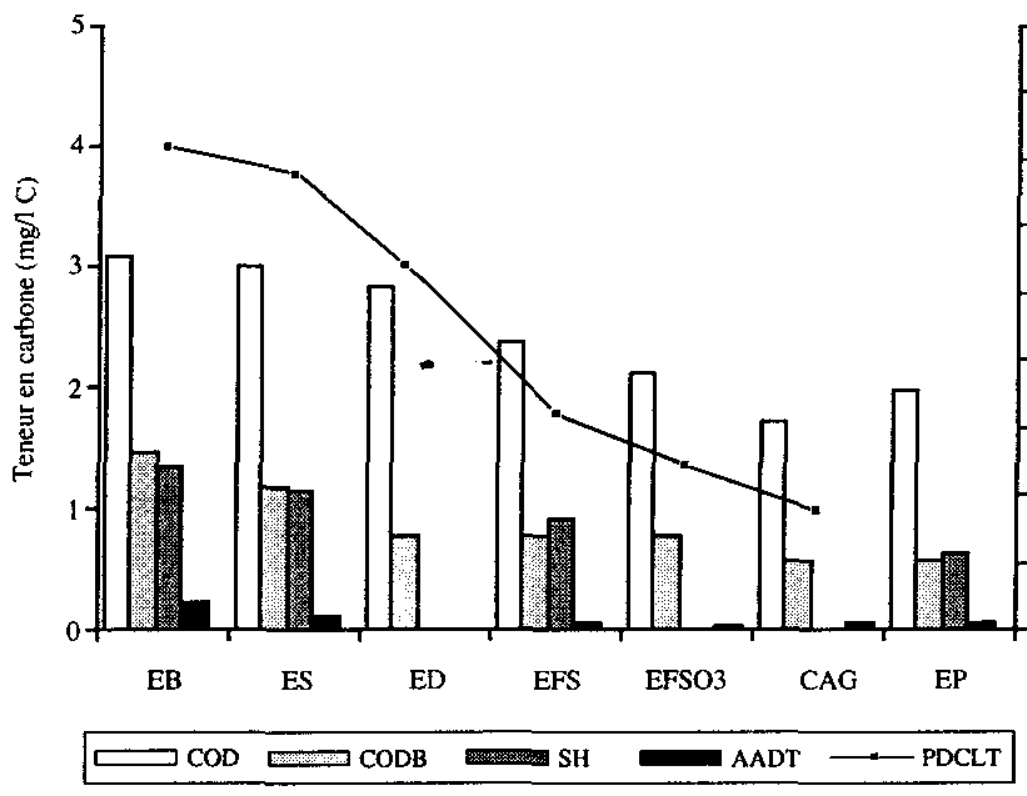

Figure 7 Evolution sur la filière de Méry-sur-Oise de la matière organique dissoute (COD, CODB, SH, AADT) et de sa demande en chlore pour le mois d'août 1993.

Evolution of dissolved organic compounds (DOC, BDOC, Humic Substances and Total Dissolved Amino Acids) and of chlorine demand potential in the water treatment plant of Méry-sur-Oise (August 1993).

CROUÉ et al., 1993). Sur l'ensemble des campagnes, l'abattement en acides aminés sur la filière, très variable, se situe entre 0 et $75 \%$ selon les saisons.

En ce qui concerne le CODB, son élimination est progressive au cours des étapes de clarification et l'abattement moyen observé à cette étape pour l'ensemble des campagnes d'analyse est de l'ordre de $43 \%$. L'ozonation induit généralement une augmentation de ce paramètre de 0,2 à $0,5 \mathrm{mg} \mathrm{l}^{-1} \mathrm{C}$ (soit des pourcentages d'augmentation de 20 à $80 \%$ du CODB de l'eau filtrée sur sable). La filtration biologique sur charbon actif en grains abaisse ensuite cette valeur de 15 à $50 \%$ en moyenne, ce qui conduit à une élimination globale sur la filière qui se situe globalement autour de 45 à $50 \%$.

\subsection{Essais de corrélation}

La grande quantité de résultats obtenus au cours de cette période d'analyses permet d'envisager des études mettant en relation les divers paramètres étudiés. En particulier, il s'avère intéressant d'examiner sur ces eaux:

- d'une part, la contribution des acides aminés dissous à la valeur du carbone organique dissous biodégradable ;

- d'autre part, l'impact de la fraction biodégradable du carbone organique dissous et de la teneur en acides aminés sur la valeur du paramètre demande en chlore. 
Dans ce but, des corrélations ont été tentées entre les différents paramètres : CODB, potentiel total de demande en chlore, acides aminés dissous totaux en regroupant l'ensemble des résultats obtenus pendant les 13 campagnes d'analyses sur les eaux brutes et en cours de traitement (fig. 8 à 10).

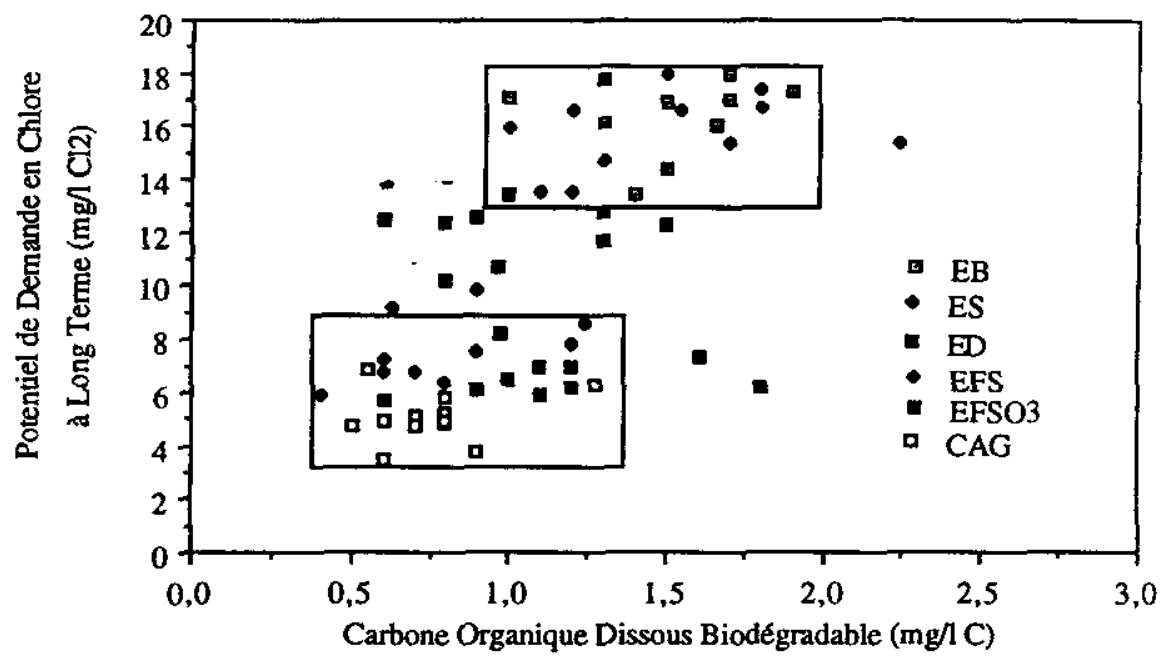

Figure 8 Tentatives de corrélation entre le potentiel de demande en chlore à long terme et le CODB pour l'ensemble des eaux de la filière.

Correlation of long-term chlorine demand versus BDOC for the different samples collected in the water treatment plant. The upper rectangle corresponds to samples from the early stages of treatment (EB, ES); the lower rectangle represents samples collected after treatment.

Notons que les acides aminés étant présents dans les eaux essentiellement sous forme combinée, ils réagissent donc lentement vis à vis du chlore, contrairement aux acides aminés libres très réactifs. C'est pour cette raison que le paramètre demande en chlore à long terme a été choisi lors des corrélations plutôt que le paramètre demande en chlore à court ou moyen terme.

En ce qui concerne la corrélation entre le CODB et le potentiel de demande en chlore (PDCLT), on peut sur la figure 8 distinguer deux zones (schématisées sur cette figure par 2 rectangles) : l'une de ces zones correspond aux eaux de début de filière (EB, ES) qui sont chargées en azote ammoniacal intervenant dans la demande en chlore et qui présentent un fort CODB, l'autre aux eaux de fin de filière, possédant un faible potentiel de demande en chlore à long terme et parallèlement les valeurs de CODB les plus basses. On peut noter aussi que mêrne en sélectionnant des eaux de fin de filière (CAG voire EFSO3), moins chargées en matières organiques, aucune corrélation n'est vérifiée.

L'analyse des figures 9 et 10 montre clairement qu'il n'existe pas de corrélation directe entre les acides aminés dissous totaux et le carbone organique dissous biodégradable d'une part (fig. 9), et le potentiel de demande en chlore à long terme, d'autre part (fig. 10). Cette absence de corrélation n'est pas particulièrement étonnante eu égard à la faible participation des acides aminés au COD des eaux. 


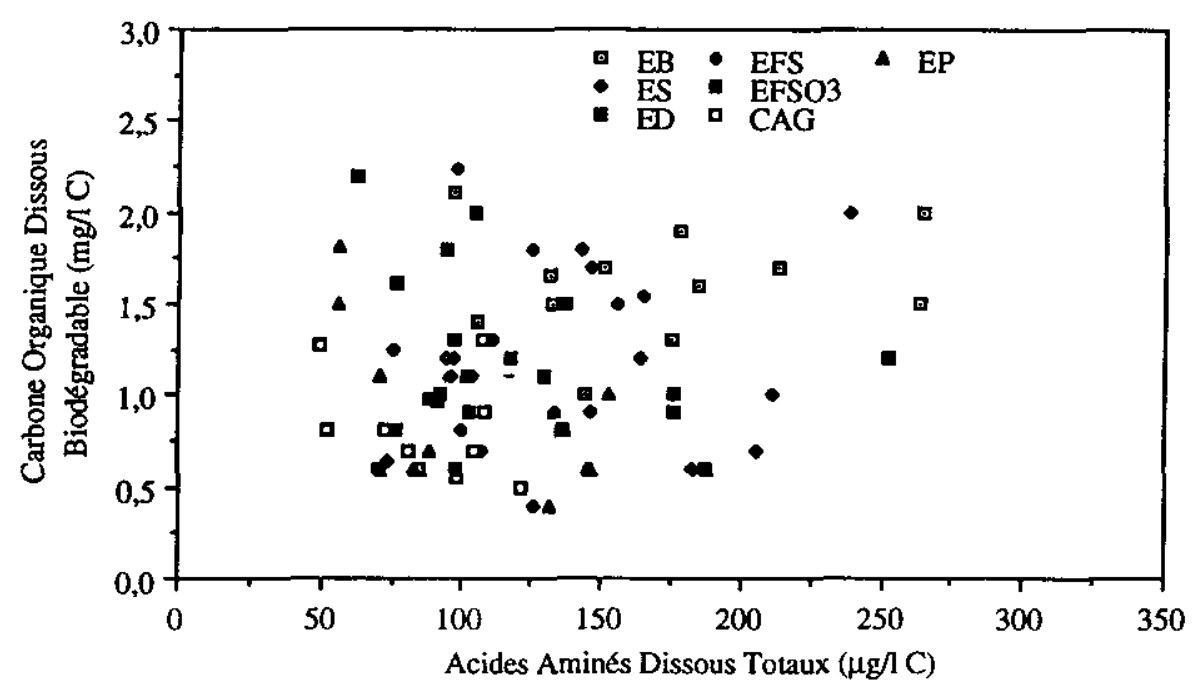

Figure 9 Tentatives de corrélation entre le CODB et la teneur en acides aminés dissous totaux pour l'ensemble des eaux de la filière.

Relationship between BDOC and amino acid content for the different samples collected in the water treatment plant. .

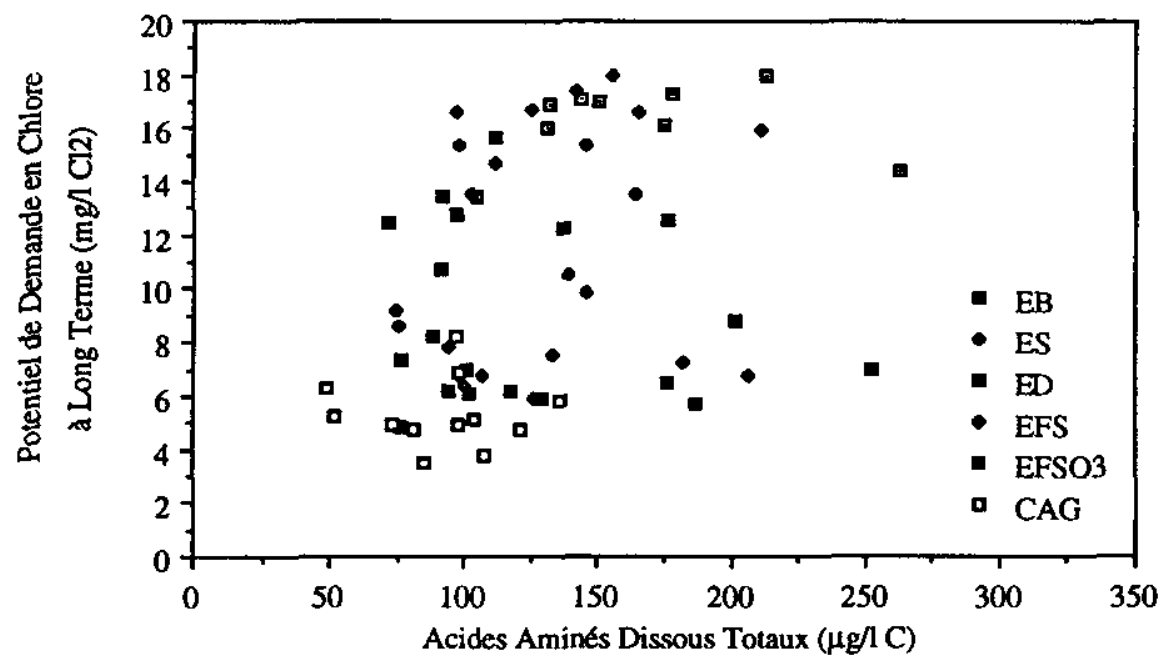

Figure 10 Tentatives de corrélation entre le potentiel de demande en chlore à long terme et la teneur en acides aminés dissous totaux, pour lensemble des eaux de la filière

Correlation of chlorine demand versus amino acid content for the different samples collected in the water treatment plant. 


\subsection{Contribution de la matière organique dissoute à la demande en chlore et au CODB}

Malgré l'absence de relation directe entre ces paramètres, il est cependant possible, en se basant sur les données bibliographiques, d'estimer la participation de cette matière organique analysée spécifiquement (substances humiques et acides aminés dissous totaux) au CODB et au potentiel de demande en chlore. Notons que les eaux de fin de filière montrent peu de différence entre le carbone organique total et sa fraction dissoute. Ceci nous permet d'envisager une relation entre le potentiel de demande en chlore et la teneur en matière organique dissoute bien que les potentiels de demande en chlore aient été déterminés sur des eaux non filtrées sur $0,45 \mu \mathrm{m}$, contrairement aux substances humiques et aux acides aminés. Les résultats issus de la bibliographie sont indiqués dans le tableau 3 ; ils servent de support aux calculs des participations au CODB et au PDCLT des acides aminés dissous totaux et des substances humiques contenus dans les eaux traitées de Méry-sur-Oise. Ces derniers calculs sont réalisés sur la base des concentrations moyennes en composés organiques spécifiques identifiés sur ces mêmes eaux au cours des différentes campagnes analytiques.

Si les substances humiques ne peuvent être que faiblement impliquées dans la valeur du CODB, les AADT peuvent apporter une contribution allant de 5

Tableau 3 Participation des acides aminés et des substances humiques au CODB et au potentiel de demande en chlore à long terme. Résultats obtenus et données bibliographiques.

Table 3 Contribution of amino acids and humic substances to BDOC and long-term chlorine demand potential. Results and bibliography.

\begin{tabular}{|c|c|c|}
\hline & Acides aminés dissous totaux & Substances humlques \\
\hline $\begin{array}{l}\text { Concentrations moyennes } \\
\text { dans les eaux traitées de Méry-sur-Oise }\end{array}$ & 50 à $150 \mu g \mathrm{r}^{-1} \mathrm{C}$ & 0,3 à $1,2 \mathrm{mg}^{-1} \mathrm{C}$ \\
\hline Participation au COD & 1,5 à $7 \%$ & 16 à $35 \%$ \\
\hline $\begin{array}{l}\text { Biodégradabilité } \\
\qquad \mathrm{CODB} / \mathrm{COO} \text { en } \%\end{array}$ & $\begin{array}{l}\text { acides aminés libres: } \\
76 \%^{\mathrm{a}} \\
85 \% \mathrm{~b} \\
\text { acides aminés combinés : } \\
63 \%^{\mathrm{a}}\end{array}$ & faiblec \\
\hline Participation au CODB calculée & $\begin{array}{l}5 \text { à } 25 \% \\
0,05 \text { à } 0,1 \mathrm{mg}^{-1} \mathrm{C}\end{array}$ & I \\
\hline $\begin{array}{l}\text { Demande en chlore } \\
\qquad \mathrm{mg} \mathrm{Cl}_{2} / \mathrm{mg} \mathrm{C}\end{array}$ & $\begin{array}{l}\text { acides aminés libres : } \\
2,5 \text { à } 16,5 \mathrm{mg} \mathrm{Cl}_{2} / \mathrm{mg} \mathrm{C}^{\mathrm{d}} \\
\text { acides aminés combinés : } \\
2,5 \text { a } 4 \mathrm{mg} \mathrm{Cl} \mathrm{Cl}_{2} / \mathrm{mg} \mathrm{C}^{\mathrm{d}}\end{array}$ & 0,8 à $2 \mathrm{mg} \mathrm{Cl} / \mathrm{mg} \mathrm{Ce}$.t \\
\hline $\begin{array}{l}\text { Participation à la demande en chlore } \\
\text { (PDCLT) calculée }\end{array}$ & $\begin{array}{l}5 \text { à } 23 \% \\
0,3 \text { à } 0,9 \mathrm{mg} \mathrm{H}^{-1} \mathrm{Cl}_{2}\end{array}$ & $\begin{array}{l}10 \text { à } 40 \% \\
0,4 \text { à } 1,7 \mathrm{mg} \mathrm{t} \mathrm{r}^{-1} \mathrm{Cl}_{2}\end{array}$ \\
\hline
\end{tabular}

AGBEKODO (1994).

- MERLET et al. (1991).

c LEENHEER et al. (1989).

ه HUREIKI (1993).

Q RECKHOW (1984).

f LEGUBE et al. (1990). 
à $25 \%$ du CODB des eaux traitées. Pour la demande en chlore, chacune des classes intervient de manière significative et leur participation globale dans l'eau produite pourrait atteindre 40 à $60 \%$ du potentiel de demande en chlore à long terme. Compte tenu du pourcentage du COD total représenté par ces composés (à savoir 20 à $35 \%$ ), cette contribution parait tout à fait notable.

Quelques réserves doivent cependant être apportées quant à ces simulations de contribution. En effet, les concentrations en AADT sur lesquelles sont basés ces calculs, sont vraisemblablement sous estimées en raison de l'étape d'hydrolyse, qui ne conduit pas à une récupération quantitative de tous les acides aminés combinés (protéines ou polypeptides), voire même libres. De plus, les données bibliographiques utilisées dans le tableau 3 pour les acides aminés se réfèrent à des molécules modèles en solution pure qui ne sont pas nécessairement représentatives des concentrations particulières et des structures, rencontrées dans les eaux naturelles. Enfin, les acides aminés présents dans les substances humiques voient leur participation à la demande en chlore comptabilisée deux fois.

\section{4- CONCLUSION}

La matière organique présente dans les eaux brutes de Méry-sur-Oise et éliminée au cours des traitements correspond en moyenne à $40 \%$ du COD, $50 \%$ du CODB, $70 \%$ du potentiel de demande en chlore à long terme et 0 à $75 \%$ des acides aminés dissous totaux.

Si la fraction biodégradable du COD semble éliminée de façon légèrement plus importante que la fraction réfractaire, et bien que les eaux qui présentent les plus fortes valeurs de CODB possèdent parallèlement les plus forts potentiels de demande en chlore, il ne semble pas exister de corrélation significative entre ces deux paramètres.

En ce qui concerne la demande en chlore, compte tenu des concentrations en substances humiques et en acides aminés dissous dans les différentes eaux, on peut estimer que leur participation globale atteindrait 40 à $60 \%$ du potentiel de demande en chlore à long terme, ce qui, comparé à la fraction de COD qu'ils représentent (20 à $35 \%$ ), met en évidence l'importance de ces paramètres pour l'optimisation de l'élimination de la demande en chlore des eaux traitées. De même, en fin de filière, si les acides aminés n'interviennent que pour 1,5 à $7 \%$ du COD des eaux traitées, leur élimination induirait un abattement du CODB et du potentiel de demande en chlore à long terme sensiblement plus important, de l'ordre de 5 à $23 \%$.

En conséquence, si les acides aminés dissous totaux n'expliquent pas à eux seuls les fortes valeurs de CODB et de demande en chlore à long terme dans les eaux traitées, leur intérêt est clairement mis en évidence. Pour optimiser les traitements et assurer le maintien de la qualité de l'eau dans les réseaux de distribution, les recherches devront se poursuivre dans cette voie et s'orienter vers d'autres classes de composés, susceptibles de participer au CODB (en particulier les carbohydrates) ou à la demande en chiore (en particulier les acides insaturés). 


\section{REMERCIEMENTS}

Les auteurs remercient le Syndicat des Eaux d'lle-de-France (SEDIF) pour le soutien financier apporté à cette étude.

\section{RÉFÉRENCES BIBLIOGRAP̄HIQUES}

AFNOR (1987). Essais des eaux : dosage du chlore libre et du chlore total par la méthode colorimétrique à la N,N-diéthylphénylène-1,4 diamine. Norme Française, NFT 90-038.

AGBEKODO M.K.A., 1994. Élimination par nanofiltration des composés organiques d'une eau de surface prétraitée - Caractérisation du cartone organique dissous avant et après nanofiltration. Thèse de Doctorat, Université de Poitiers.

CRANE M.A., KOVACIC P., KOVACIC D.E., 1980. Volatile halocarbon production from the chlorination of marine algal byproducts including D-mannitol, Environ. Sci. Tectinol., 14(11), 1371-1374.

CROUÉ J.P., LEFEBVRE E., MARTIN B., LEGUBE B., 1993. Removal of dissolved hydrophobic and hydrophilic organic substances during coagulationflocculation of surface waters, Wat. Sci. Technol., 27, 143-152.

DOSSIER BERNE F., PANAIS B., MERLET N., CAUCHI B., LEGUBE 8., 1994a. Analyse des acides aminés dissous totaux dans les eaux à potabiliser en cours de traitement, Environmental Technology, 15(10), 901-916.

DOSSIER BERNE F., PANAIS B., MERLET N., JADAS-HÉCART A., CAUCHI B. et LEGUBE B., 1994b. Automatisation de la demande en chlore à long terme d'eaux traitées. Acceptée pour publication dans Wat. Res.

EL MORER A., 1992. Contribution à l'étude cinétique de la chloration des acides aminés libres et combinés. Thèse de 3ème cycle, Université de Tétouan, Maroc.

HUREIKI L., 1993. Étude de la chloration et de rozonation d'acides aminés libres et combinés en milieu aqueux dilué. Thèse de Doctorat, Université de Poitiers.

JADAS-HÉCART A., 1989. Contribution à l'étude de la demande en chlore à long terme d'une eau potable - Modélisation et identification des précurseurs organiques. Thèse de Doctorat, Université de Poitiers.

JADAS-HÉCART A., EL MORER A., STITOU M., BOUILLOT P., LEGUBE $B$., 1992. Modélisation de la demande en chlore d'une eau traitée, Wat. Res., 26(8), 1073-1084.

JORET J.C., LÉVI Y., 1986. Méthode rapide de détermination du carbone éliminable des eaux par voie biologique, Tribune du Cebedeau, 39(510), 3-9.

LE ClOIREC C., LE CLOIREC P., MORVAN J., MARTIN G., 1983. Formes de l'azote organique dans les eaux de surface : brutes ou en cours de potabilisation, Revue Française des Sciences de I'Eau, 2, 25-39.

LEENHEER J.A., MCKNIGHT D.M., THURMAN E.M., MacCARTHY P., 1989. Structural components and proposed stuctural models of fulvic acid from the Suwannee river, In "Humic Substances in the Suwannee river, Georgia Interactions, properties, and proposed structurs ", U.S. Geological Survey Open-File Report 87557, 335-359.

LEFEBVRE E., LEGUBE B., 1990. Coagulation par $\mathrm{Fe}$ (III) de substances humiques extraites d'eaux de surface : effet du $\mathrm{pH}$ et de la concentration en substances humiques, Wat. Res., 24(5), 591-606.

LEGUBE B., XIONG F., CROUÉ J.P., DORÉ M., 1990. Étude sur les acides fulviques extraits d'eaux superficielles 
françaises (extraction, caractérisation et réactivité avec le chlore), Revue des Sciences de l'Eau, 3, 399-424.

MERLET N., MERLET Y., PRÉVOST M., DESJARDINS R., BABLON G., 1991. Removal of organic matter in BAC filters : the link between BDOC and chlorine demand, AWWA Water Quality Technology Conference, Orlando, Floride.

RECKHOW D.A., 1984. Organic halide formation and the use of preozonation "and alumn coagulation to control organic halide precursors, $P h$. D. Thesis, Depart- ment of Environmental Sciences and Engineering, CHAPEL HILL (N.C).

ROTH E.S., MAIER D., 1991. Biodegradation of amino acids as a new bacteria toxicity test, AWWA Water Quality Technology Conference, Orlando, Floride.

THURMAN E.M., 1985. Developments in biochemistry: organic geochemistry of natural waters, M. NIJHOFF, Dr W. JUNK Publishers, DORDRECHT.

THURMAN E.M., MALCOLM R.L., 1981. Preparative isolation of aquatic humic substances, Environ. Sci. Technol., 15, 463-466. 